\title{
Stereotactic Body Radiation Therapy (SBRT) for Clinically Localized Prostate Cancer Utilizing the Elekta Infinity Linac System in a Community Setting
}

\author{
Farber LA* and Speck T \\ Section Chief of Radiation Oncology and Senior Medical Director, USA
}

Submission: June 15, 2018; Published: July 03, 2018

"Correspondence Address: Leonard Farber, Section Chief of Radiation Oncology and Senior Medical Director, USA, Tel: +1 212-300-0663; Email: lafarbermd@gmail.com

\begin{abstract}
Introduction: Hypofractionated, stereotactic body radiotherapy (SBRT) is an evolving therapy for the treatment of localized prostate cancer. We present our experience using the Elekta Infinity Linac system with SBRT for prostate cancer patients in the community setting.

Materials and Methods: Our center performed a retrospective review of our database of patients undergoing SBRT for prostate cancer since initiation of the program in 2011.All patients were treated on the Elekta Infinity system.The prescription dose was 35 Gy in five fractions.

Results: 41 patients were reviewed for this analysis.According to NCCN criteria 15 were low-risk, 12 intermediate-risk, and 13 highrisk.52.5\% of patients were treated with static field (95.3\% 7-field and $4.7 \% 5$-field) beam arrangements.47.5\% of patients were treated with VMAT.Overall time on table was $33.28 \mathrm{~min}$ for static beams and $32.31 \mathrm{~min}$ for VMAT.Overall beam on time was $10 \mathrm{~min}, 14 \mathrm{~min}$, and $8 \mathrm{~min}$ for 5 -field static beams, 7-field static beams, and VMAT, respectively.32 patients were compliant with obtaining follow up PSAs.Biochemical response regarding PSA demonstrated all patients except one to have a significant response, and all patients out one year from SBRT demonstrated a PSA within normal range with duration out to four years to date.
\end{abstract}

Conclusion: SBRT is a feasible and reproducible treatment option for patients with clinically localized prostate cancer in the community setting.Logistical challenges to standard 9-week fractionation treatment as well as access to centers exclusive to SBRT demonstrate that treatment of prostate cancer with SBRT on linac-based systems such as the Elekta Infinity warrant further consideration.

\section{Introduction}

Stereotactic body radiotherapy (SBRT) has been an emerging technology for treatment of prostate cancer.Utilizing data from other hypofractionated techniques including external beam radiation therapy and high dose rate (HDR) brachytherapy, SBRT has been used with increasing frequency [1-6].Evidence from clinical studies using hypofractionated radiation has demonstrated that the $\alpha / \beta$ ratio for prostate cancer is lower than for that of the bladder and rectum, and therefore using fewer and higher dose per fraction deliver, a therapeutic gain can be achieved [8,9].Similarly, HDR brachytherapy delivers large doses per fraction and has been used at institutions for low, intermediate, and high-risk patients without the addition of androgen deprivation therapy (ADT) and with favorable outcome comparable to standard fraction radiation therapy [10-12].These studies further demonstrated that the addition of ADT failed to improve outcome with dose-escalated RT in the intermediateand high-risk patient population [10].SBRT takes advantage of the prostate's low $\alpha / \beta$ ratio similar to HDR brachytherapy and like other external beam radiation techniques, does so in a noninvasive approach.

Most of clinical reports have come from the use of CyberKnife system (Accuray Inc., Sunnyvale, CA, USA).Initial reports from examined outcome data for low risk as primary treatment $[5,13$ 17] and as a boost [18] and subsequently for intermediate and high-risk patients [19].Katz reported five-year outcome data for low-, intermediate- and high-risk patients utilizing CyberKnife and demonstrated that at a median follow-up of 50 months the 5-year freedom from biochemical failure was 97\%, 93\%, and $74 \%$, respectively.Late RTOG toxicity was mild for GI and GU complications.Mean EPIC urinary and bowel QOL declined at 1-month post-treatment and returned to baseline by year $2.76 \%$ of patients potent at baseline remained potent [19]. Other studies demonstrate comparable results $[1,2,14,20]$ using doses of 35-36.25 Gy in five fractions. 


\section{Cancer Therapy \& Oncology International Journal}

Given the outcome data and toxicity profile for SBRT for prostate cancer, logistical considerations such as access to CyberKnife, and the required tracking for the prolonged beamon time we explored the feasibility and practical application of SBRT using a linac based system, the Elekta Infinity (Elekta AB Stockholm, Sweden).This study reports our clinical experience with SBRT with the Elekta Infinity for a range of patients.

\section{Materials and Methods}

\section{Patient Selection}

Patients eligible for treatment inclusion had histologicallyconfirmed clinically localized adenocarcinoma of the prostate. Exclusion criteria included involved lymph nodes or distant metastases on imaging and/or prior pelvic radiotherapy.All patients were offered standard 9-week radiation therapy and patients with unfavorable intermediate-risk and all high-risk patients were offered ADT.All patients refused all standard of care treatment options.The initial patient population included low-risk and favorable intermediate-risk patients and was expanded to included unfavorable intermediate-risk and highrisk patients.Two patients underwent placement of hydrogel perineal spacers prior to treatment planning.All patients underwent an extent of disease workup as outline in the NCCN Guidelines (National Comprehensive Cancer Network).

\section{SBRT Treatment Planning}

All patients underwent a CT/Simulation using the Toshiba Aquilion large bore CT/Simulator (Toshiba America Medical Systems, Inc, Tustin, CA, USA).30 minutes prior to the CT the bladder is emptied and patients subsequently were instructed to drink 32 ounces of water.Patients were positioned supine on the simulation table. $2 \mathrm{~mm}$ thin-cut images were obtained of the pelvis starting beneath the penile bulb and extending to above the bladder.All patients had placement of an endorectal balloon (ERB) for prostate immobilization following Fleets enema and data recorded for reproducibility.Body immobilization was achieved with the Civco Pro-Lok SBRT system (Civco Medical Solutions, Coralville, IA, USA).A Vac-Lok was utilized to immobilize the patients' legs. Using the scout image, the approximate level of the midprostate was demarcated and the 0 point determined. The patients were marked and radioopaque markers were placed externally.

Patients underwent an MRI with ERB placement either the same day or within 24 hours of CT/Simulation.CT/MRI fusion was performed on all cases, initially with Elekta CMS Monaco planning and subsequently with MIM Maestro (MIM Software, Cleveland, OH).MRI image fusion included T1 pre- and postgadolinium VIBE, and T2.The clinical target volume (CTV) included the prostate and $1 \mathrm{~cm}$ of proximal seminal vesicles for low- and intermediate-risk patients and the prostate and entire seminal vesicles for high-risk patients. The planning treatment volume (PTV) was an expansion of $0.8 \mathrm{~cm}$ superiorly, $0.6 \mathrm{~cm}$ inferiorly, $0.7 \mathrm{~cm}$ left and right, $0.8 \mathrm{~cm}$ anteriorly, and $0.6 \mathrm{~cm}$ posteriorly.The prescription dose was 35 Gy to the PTV in five fractions of 7 Gy each. $95 \%$ of the PTV received $100 \%$ of the prescribed dose.Hot spots were placed in MRI areas of high risk or based on biopsy reports of tumor localization.

Organs at risk (OAR) including the rectum, bladder, and penile bulb were demarcated and dose constraints were applied based on published data [21-24].Monte Carlo based treatment planning was utilized with the Elekta CMS Monaco planning system. The Monaco system incorporates a two-stage IMRT optimization procedure and a version of the XVMC Monte Carlo dose calculation algorithm. The fluence distribution is optimized using the hard constraints for organs at risk.In the second stage, the beams are segmented and their weights optimized while maintaining the results of the first phase.QA is performed using a static coronal field and a diode array device MapCHECK (Sun Nuclear Corp, Melbourne, FL, USA).A criterion of 95\% passing with $3 \%$ agreement, $3 \mathrm{~mm}$ DTA with a $10 \%$ threshold was met on most cases.Given the resolution of CBCT imaging on the Elekta Infinity gold fiducial markers were not utilized.All patients underwent a treatment verification utilizing cone beam CT (CBCT) with an endorectal balloon and all immobilization set at CT/Simulation prior to the onset of radiation therapy(Figures $1 \& 2$ ).

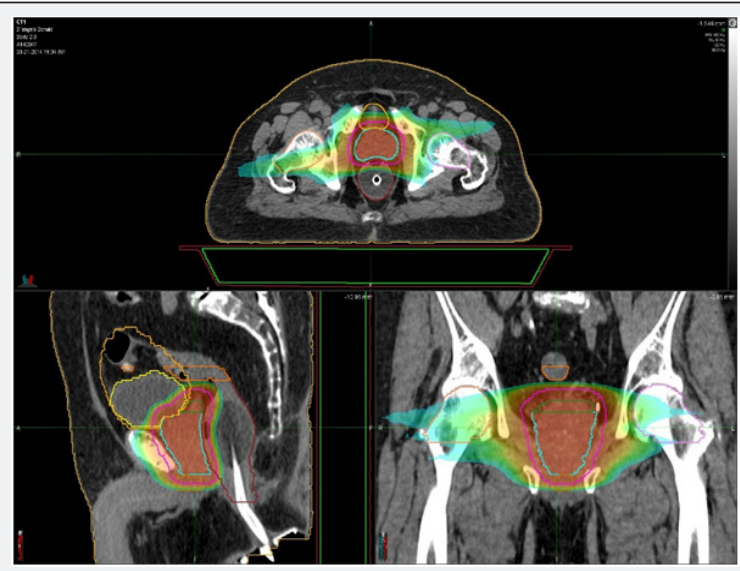

Figure 1: Sample SBRT Treatment PlanColorwash-represented isodose lines as follows: $110 \%$ of the prescription dose, brown; $100 \%$ of the prescription dose red; $75 \%$ of prescription dose, yellow; $50 \%$ of prescription dose, blue. 


\section{Cancer Therapy \& Oncology International Journal}

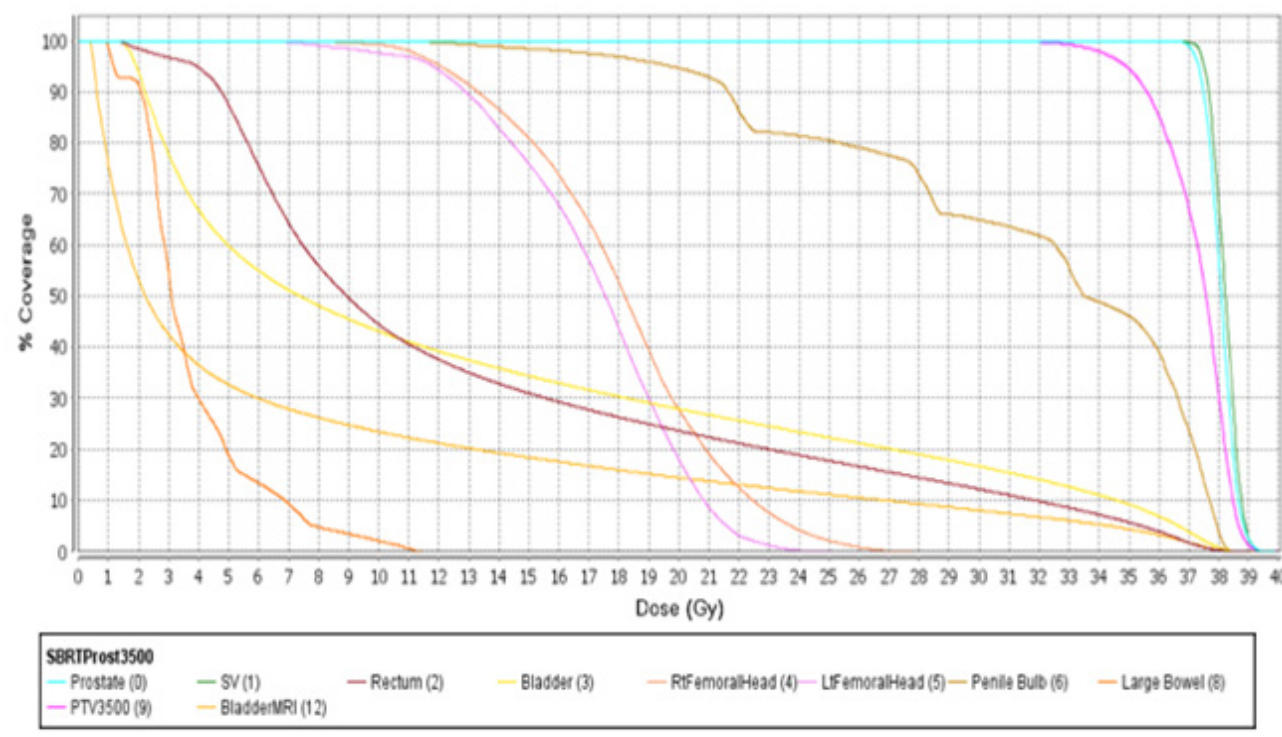

Figure 2: Sample DVH for SBRT Prostate Patient

\section{SBRT Treatment Delivery}

Patients were treated in five consecutive days unless interruption occurred for medical reasons.An endorectal balloon was placed and inflation matched to planning records.A pre-treatment CBCT was obtained in counterclockwise rotation. The planning CT computer overlay on the CBCT image was then matched for positioning utilizing the HexaPOD evo RT table system (Elekta AB Stockholm, Sweden).The HexaPOD evo RT System was guided by linac room infrared-camera cameras. Sub-millimeter patient positioning accuracy was obtain in six degrees of freedom, correcting for translation errors $(\mathrm{x}, \mathrm{y}, \mathrm{z})$, as well as all rotational errors (roll, pitch, and yaw).Table shifts were automatically applied.Treatment was then performed with an arc utilizing volumetric arc therapy (VMAT) in a clockwise position (initially, multiple field static beams were utilized and subsequently VMAT when it became available).A second CBCT was obtained in counterclockwise rotation and Hexapod shifts obtained and applied.A second VMAT arc was performed.Typical arcs were 300 degrees on output of $1 \mathrm{~cm}$ micro leaf collimators (MLC).During treatment real-time external anatomy movement was monitored by C-RAD Sentinel, utilizing patient contoured laser topography (C-RAD AB Uppsala, Sweden).This allowed for intra-fraction motion detection using the patient's external anatomy without surrogate devices. $3 \mathrm{~mm}$ maximum deviation thresholds were set and therapists continuously monitored the real-time display.Selected patients underwent a third CBCT after treatment delivery.

\section{Results}

From March 2011 to May 2014, 41 prostate cancer patients were treated with our SBRT protocol at our center.Patient characteristics are indicated in Table 1.The average patient age was 67 years (range 48-81 years).Gleason score was $<6$ in 18 (45\%) patients, 7 in 14 (35\%) patients, 8 in $6(15 \%)$ patients, and 9 in $2(5 \%)$ patients.Using NCCN guidelines 15 (37.5\%) were low-risk, 12 (30.0\%) were intermediate-risk, and 13 (32.5\%) were high-risk.Most patients (97.5\%) did not receive ADT.
Table 1:Patient Characteristics.

\begin{tabular}{|c|c|}
\hline Age & $\mathbf{6 7}(\mathbf{4 8 - 8 1})$ \\
\hline Pre-Treatment PSA (ng/mL) & $6.32(2.53-429)$ \\
\hline Prostate Size $\left(\mathrm{cc}^{3}\right)$ & $69.2(36.03-157.29)$ \\
\hline Gleason Score & $18(45 \%)$ \\
\hline$\leq 6$ & $14(35 \%)$ \\
\hline 7 & $6(15 \%)$ \\
\hline 8 & $2(5 \%)$ \\
\hline 9 & $15(37.5 \%)$ \\
\hline NCCN Risk Category & $12(30.0 \%)$ \\
\hline Low & $13(32.5 \%)$ \\
\hline Intermediate & $17(37.5 \%)$ \\
\hline High & $10(25 \%)$ \\
\hline Stage Grouping & $8(22.5 \%)$ \\
\hline I & $5(12.5 \%)$ \\
\hline IIA & \\
\hline IIB & $1(2.5 \%)$ \\
\hline III & $39(97.5 \%)$ \\
\hline ADT Treatment & \\
\hline Yes & \\
\hline No & \\
\hline
\end{tabular}

Table 2: BeamCharacteristics.

\begin{tabular}{|c|c|}
\hline Field Arrangement & \\
\hline Static & $21(52.5 \%)$ \\
\hline 5-fields & $1(4.7 \%)$ \\
\hline 7-fields & $20(95.3 \%)$ \\
\hline VMAT & $19(47.5 \%)$ \\
\hline
\end{tabular}




\section{Cancer Therapy \& Oncology International Journal}

\begin{tabular}{|c|c|}
\hline MU/Fraction & \\
\hline Static & 2654.99 \\
\hline 5-fields & $2466.22(2117.59-2928.27)$ \\
\hline 7-fields & $2979.46(2541-3841.91)$ \\
\hline VMAT (2 Arcs) & \\
\hline Overall Time on Table & 33.28 min (mode 31 min) \\
\hline Static & 32.31 min (mode 29 min) \\
\hline VMAT & \\
\hline 0verall Beam on Time (BOT) & 10 min \\
\hline Static & 14 min \\
\hline 5-fields & 8 min ( 4 min/arc) \\
\hline 7-fields & \\
\hline VMAT (2 Arcs) & \\
\hline
\end{tabular}

Beam arrangements (static field versus VMAT), Monitor Units (MU) per fraction, beam-on time, and total time on table (included 2 CBCT and HexaPOD image capture and shifts) are shown in Table 2.Total time on the table defined by site set up affirmation until the initial of last beam for the static fields and for the VMAT cases until the end of the second arc.For the static fields the last field time approximated the prior utilized field and for the the VMAT, the second arc time approximated the initial arc as planning demonstrated negligible time differences.One course was utilized per patient as the differences were minor and represented only biological variations, e.g., bladder filling.52.5\% of patients were treated with static field arrangements, of those 95.3\% were 7 -field and $4.7 \%$ were 5 -field beam arrangements. All patients were subsequently treated with VMAT $(47.5 \%)$ when it became available.Overall time on table was $33.28 \mathrm{~min}$ for static beams and $32.31 \mathrm{~min}$ for VMAT.Overall beam on time was $10 \mathrm{~min}, 14 \mathrm{~min}$ and $8 \mathrm{~min}$ for 5 -field static beams, 7-field static beams, and VMAT, respectively.

32 patients were compliant with obtaining follow up PSAs. Initial PSA results were obtained beginning at 3 or 6 months from completion of SBRT, depending on patient compliance and referring urologist and were requested at 6-month intervals.PSA values grouped according to time from completion are shown in Table 3.33 patients were compliant with obtaining follow up PSAs.For all patients out one year from completion of SBRT a significant biochemical response was demonstrated, with PSA in normal range. For patients out less than one year, all, except for one, were noted to have a significant decrease in PSA.At 3-6 months one patient had a PSA at 5.1, which was down from preSBRT value of 16.3.At 6-12 months one patient had a PSA of 11.5, which was down from 14.31.The one patient with post-SBRT rise in PSA had a negative metastatic workup prior to RT with radiographic findings consistent with Paget-like bone disease.
Table 3: PSA (ng/mL) from Completion of SBRT

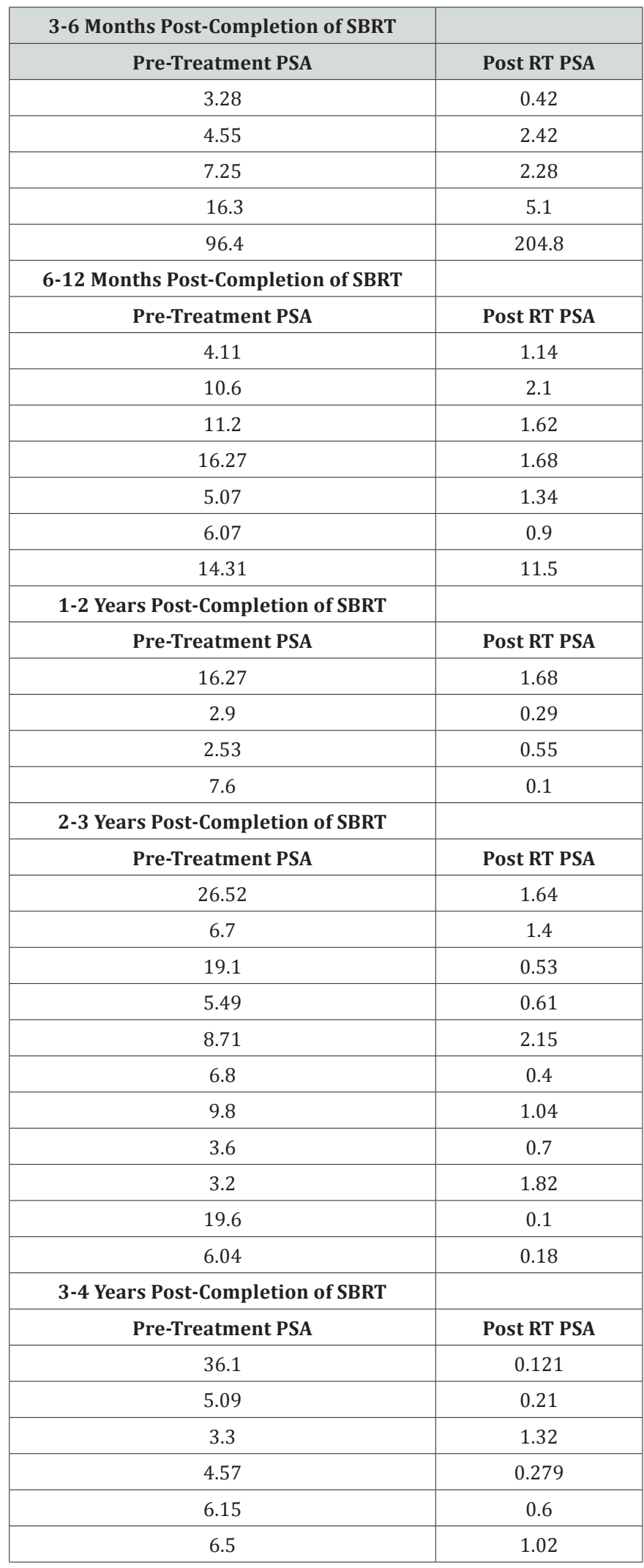

\section{Discussion}

This report demonstrates that SBRT can be performed accurately and with precise reproducibility in the community 
setting given strict planning and treatment parameters.A variety of techniques contribute to the reproducibility factors, including immobilization, planning, high resolution imaging on the linacs, intrafraction and interfraction motion monitoring.Quality assurance programs must be in place and carefully implemented.

External contour laser topography for intrafraction monitoring as acquired by C-RAD has limitations as the actual organ movement is not recorded.For this reason, it was felt that an endorectal balloon for prostate immobilization, coupled with the rapid beam on time with VMAT on the Elekta Infinity would minimize geographical miss.Our study demonstrates that this is achievable and with a high degree of confidence based on mid treatment and post treatment CBCT shifts and recorded C-RAD tolerance values.Furthermore, barring bladder filling issues, patients were able to be treated within a standard fractionation time slot.Another challenge on linac-based planning is utilization of standard $1 \mathrm{~cm}$ MLC to be able to deliver adequate dose while respective organs at risk (OAR).Utilizing modest PTV margins our planning system was able to deliver the required dose and still respect tolerance doses of OAR based on Quantec data in a timely manner.Visualization of the endorectal balloon, which abuts the posterior aspect of the prostate, adds an additional verification structure.With short beam-on times of less than $10 \mathrm{~min}$, there may be an additional benefit to the patient, with resultant decrease in patient exposure to radiation, patient comfort, and therefore, treatment reproducibility.

The literature demonstrates disease control and toxicity outcomes with SBRT as being favorable compared to conventional external beam regimens and brachytherapy $[14,15,20]$.Initial reports were shown for low-risk patients and have continued to demonstrate similar results for intermediate- and high-risk patients $[19,25]$. Furthermore, these results have been upheld for patients who have undergone SBRT without the addition of ADT [10].In the community setting, these considerations are of utmost important to present all treatment options to patients, as in our patient population for this study, all unfavorable intermediate-risk and all high-risk patients refused ADT. Our outcome data for our entire cohort of patients looking at biochemical response and toxicity profiles are currently being collected and analyzed and will be presented in subsequent publication.

\section{Conclusion}

SBRT may be a feasible and reproducible treatment option for patients with clinically localized prostate cancer.Further analysis of toxicity profile will further assess the feasibility of this treatment.Reported PSA results have suggested a biochemical response at least equivalent to other standard radiation therapy approaches.GU and GI toxicity data are currently being collected using EPIC surveys.Logistical challenges for patients to standard 9-week fractionation treatment as well as patient access to centers exclusive to SBRT demonstrate that treatment of prostate cancer with SBRT on linac-based systems such as the Elekta
Infinity warrant further consideration.It offers a treatment option for patients who may otherwise forego treatment if a patient cannot undergo 9-weeks of treatment due to travel considerations, work routines, and overall cost implications. Further outcome data will lend invaluable information regarding this modality.

\section{Acknowledgement}

We acknowledge Wesley Pedone for his participation of data collection and data analysis.

\section{References}

1. Aluwini S, van Rooij P, Hoogeman M, Bangma C, Kirkels WJ, et al. (2010) CyberKnife stereotactic radiotherapy as monotherapy for lowto intermediate-stage prostate cancer: early experience, feasibility, and tolerance. J Endourol 24(5): 865-869.

2. Friedland JL, Freeman DE, Masterson-McGary ME, Spellberg DM (2009) Stereotactic body radiotherapy: an emerging treatment approach for localized prostate cancer. Technol Cancer Res Treat 8(5): 387-392.

3. Fuller DB, Naitoh J, Lee C, Hardy S, Jin H (2008) Virtual HDR(SM) CyberKnife Treatment for Localized Prostatic Carcinoma: Dosimetry Comparison with HDR Brachytherapy and Preliminary Clinical Observations. Int J Radiat Oncol Biol Phys 70(5): 1588-1597.

4. Katz AJ, Santoro M, Ashley R, Diblasio F, Witten M (2010) Stereotactic body radiotherapy for organ-confined prostate cancer. BMC Urol 10: 1 .

5. King CR, Brooks JD, Gill H, Pawlicki T, Cotrutz C, et al. (2009) Stereotactic body radiotherapy for localized prostate cancer: interim results of a prospective phase II clinical trial. Int J Radiat Oncol Biol Phys 73(4): 1043-1048.

6. Madsen BL, Hsi RA, Pham HT, Fowler JF, Esagui L, et al. (2007) Stereotactic

7. Townsend NC, Huth BJ, Ding W, Garber B, Mooreville M, et al. (2010) Acute toxicity after CyberKnife-delivered hypofractionated radiotherapy for treatment of prostate cancer. Am J Clin Oncol 34(1): 6-10.

8. Dasu A (2007) Is the alpha/beta value for prostate tumours low enough to be safely used in clinical trials? Clin Oncol (R Coll Radiol) 19(5): 289-301.

9. Macias V, Biete A (2009) Hypofractionated radiotherapy for localised prostate cancer. Review of clinical trials. Clin Transl Oncol 11(7): 437445.

10. Krauss, D, Kestin L, Ye H, Brabbins, D, Ghilezan M, et al. (2011) Lack of Benefit for The Addition Of Androgen Deprivation Therapy To DoseEscalated Radiotherapy In The Treatment Of Intermediate- And HighRisk Prostate Cancer. Int J Radiat Oncol Biol Phys 80(4): 1064-1071.

11. Galalae R, Martinez A, Mate T, Mitchell C, Edmundson G, et al. (2004) Long-Term Outcome By Risk Factors Using Conformal High- DoseRate Brachytherapy (HDR-BT) Boost With Or Without Neoadjuvant Androgen Suppression For Localized Prostate Cancer. Int J Radiat Oncol Biol Phys 58(4): 1048-1055. 


\section{Cancer Therapy \& Oncology International Journal}

12. Martinez A, Demanes J, Vargas C, Schour L, Ghilezan M, et al. (2009) High-Dose-Rate Prostate Brachytherapy: An Excellent AcceleratedHypofractionated Treatment for Favorable Prostate Cancer. Am J Clin Oncol 33(5): 481-488.

13. Friedland JL, Freeman DE, Masterson-McGary ME, Spellberg DM (2009) Stereotactic body radiotherapy: an emerging treatment approach for localized prostate cancer. Technol Cancer Res Treat 8(5): 387-392.

14. Katz AJ, Santoro M, Ashley R, Diblasio F, Witten M (2010) Stereotactic body radiotherapy for organ-confined prostate cancer. BMC Urol 10: 1.

15. Katz A, Santoro M (2009) CyberKnife radiosurgery for early carcinoma of the prostate: A three year experience. Int J Radiat Oncol Biol Phys 75: S312-S313.

16. CyberKnife Radiosurgery for Low \& Intermediate Risk Prostate Cancer: Emulating HDR Brachytherapy Dosimetry, US National Library of Medicine, USA.

17. Freeman DE, Friedland JL, Masterson-McGary ME (2010) Stereotactic Radiosurgery for Low-Intermediate Risk Prostate Cancer: An Emerging Treatment Approach. Am J Clin Oncol 33,208.

18. Katz, A, Santoro, M, Ashley, R, Diblasio, F, Witten, M. Stereotactic Body Radiotherapy as Boost for Organ-Confined Prostate Cancer. Technol Cancer Res Treat 9: 575-582

19. Katz AJ, Santoro M, Diblasio F, Ashley R (2013) Stereotactic body radiotherapy for localized prostate cancer: disease control and quality of life at 6 years. Radiat Oncol 8: 118.
20. Freeman, DE, King CR (2011) Stereotactic body radiotherapy for lowrisk prostate cancer: five-year outcomes. Radiation Oncology 6: 3 .

21. Timmerman RD (2008) An Overview Of Hypofractionation And Introduction To This Issue Of Seminars In Radiation Oncology. Semin Radiat Oncol 18: 215-222.

22. Boike TP, Lotan Y, Cho LC, Brindle J, DeRose P, Timmerman, R. Phase I Dose-Escalation Study of Stereotactic Body Radiation Therapy for Low- and Intermediate-Risk Prostate Cancer. J Clin Oncol 29: 20202026.

23. Kim DW, Cho LC, Straka C, Christie A, Lotan Y, et al. (2014) Predictors of Rectal Tolerance Observed in a Dose-Escalated Phase 1-2 Trial of Stereotactic Body Radiation Therapy for Prostate Cancer. Int J Radiation Oncol Biol Phys 89(3): 509e517.

24. Alongi F, Cozzi L, Arcangeli S, Iftode C, Comito T, et al. (2013) Linac based SBRT for prostate cancer in 5 fractions with VMAT and flattening filter free beams: preliminary report of a phase II study. Radiat Oncol 8: 171.

25. Vu CC, Haas JA, Katz AE, Witten MR (2014) Prostate-specific antigen bounce following stereotactic body radiation therapy for prostate cancer. Front Oncol 4: 8.

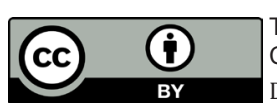

This work is licensed under Creative Commons Attribution 4.0 License

DOI: 10.19080/CTOIJ.2018.11.555811 